\title{
Leiomyosarcoma of the inferior vena cava. Our experience and a review of the literature
}

\author{
CRISTIAN Bogdan RUSU ${ }^{1)}$, LILIAN GORBATÂI ${ }^{2)}$, LUdOVIC SZATMARI ${ }^{3)}$, RUMELIA KOREN ${ }^{4}$, \\ CĂTĂLINA ILEANA BUNGĂRDEAN ${ }^{5)}$, BOGDAN OVIDIU FECICHE ${ }^{1)}$, CĂLIN BUMBULUT' ${ }^{6)}$, \\ IULIA MĂDĂLINA ANDRAŞ ${ }^{7,8)}$, RĂZVAN RAHOTĂ ${ }^{8)}$, TEODORA TELECAN ${ }^{7}$ ), IOAN COMAN ${ }^{7,8)}$, \\ LEA RATH-WOLFSON ${ }^{4}$, NICOLAE CRIŞAN ${ }^{7,8)}$
}

\author{
1) Department of Urology, Satu Mare Emergency County Hospital, Satu Mare, Romania, affiliated with \\ Iuliu Hatieganu University of Medicine and Pharmacy, Cluj-Napoca, Romania \\ 2) Department of Intensive Care, Satu Mare Emergency County Hospital, Satu Mare, Romania \\ 3) Department of Pediatric Surgery, Satu Mare Emergency County Hospital, Satu Mare, Romania \\ 4) Department of Pathology, Hasharon Hospital, Rabin Medical Center, Petach Tikva, affiliated with \\ Sackler Faculty of Medicine, Tel Aviv University, Tel Aviv, Israel \\ ${ }^{5)}$ Department of Pathology, Municipal Clinical Hospital, Cluj-Napoca, Romania \\ 6) GP Medical Civil Society, Bobocului, Satu Mare, Romania \\ 7) Department of Urology, Iuliu Hatieganu University of Medicine and Pharmacy, Cluj-Napoca, Romania \\ ${ }^{8)}$ Department of Urology, Municipal Clinical Hospital, Cluj-Napoca, Romania
}

\begin{abstract}
Leiomyosarcoma (LMS) of the inferior vena cava (IVC) is a rare malignant tumor, accounting for $2 \%$ of all LMSs. Less than 400 cases have been reported in literature. Computed tomography (CT) is the most accurate imaging method in assessing the location of the tumor within the IVC and magnetic resonance imaging (MRI) accurately identifies its extent and the potential for surgical resection. We present the case of a patient with inferior vena cava leiomyosarcoma (IVCL), for whom the pathological diagnosis was different from the initially expected one, the tumor appearance on pre-operative imaging mimicking renal cell carcinoma. The intraoperative difficulty of approaching renal hilum and IVC was a factor suggesting the vascular origin of the tumor, which was confirmed at pathological analysis. The extensive defect in the IVC after tumor excision led to the decision of complete transverse suturing of IVC, as significant collateral venous circulation was already present. Because IVCL is a rare disease, there is scarce data regarding the prognosis and treatment options. Long-term survival depends on the extent of the surgery. The need of vascular reconstruction is not always mandatory. Despite high recurrence rates, no consensus regarding adjuvant treatment exists yet. A multidisciplinary approach including surgical oncologists and vascular surgeons is mandatory to achieve the best patient outcomes. Perioperative planning, coordination and adherence to oncological techniques are critical.
\end{abstract}

Keywords: leiomyosarcoma, inferior vena cava, long-term survival, vascular reconstruction.

\section{Introduction}

Leiomyosarcoma (LMS) of the inferior vena cava (IVC) is a rare malignant tumor emerging from the smooth muscle cells located in the middle layer of the venous wall, with intra- or extra-luminal growth [1], accounting for $2 \%$ of all LMSs [2] and occurring most frequently in middle-aged women [3]. Since Perl \& Virchow's first description in 1871 [4], less than 400 cases have been reported in literature [5].

The tumor arises from the tunica media of the blood vessels and can grow either intraluminal, extraluminally or mixed, with one of the patterns being predominant, although the extraluminally type has bed reported as the most frequent [6]. The lesion is included into one of three levels depending on the relation with the hepatic and renal veins, respectively. Zone I represents the infrarenal IVC, zone II the IVC between the hepatic and the renal veins, and zone III the segment above the hepatic veins and up to the right atrium [7], the inferior vena cava leiomyosarcoma (IVCL) most frequently occurring in the middle segment. The tumor can infiltrate both hepatic and renal vessels.
Accurate diagnosis of IVCL needs histological confirmation. The three defining histopathological traits that may predict tumoral behavior (leaning towards a benign or an aggressive pattern) and the overall prognosis are: tumor differentiation, mitotic index and proportion of necrosis [8]. However, these scores may be subjected to errors, as described by Taylor et al. [9], where a mitotic index as low as 2 did not anticipate the rapid tumoral progression of the presented case. Two factors would explain the poor prognosis: the tumoral localization and the low degree of tumoral differentiation [10].

IVCL is a malignant slow-growing tumor that produces late clinical manifestations. While upper segment tumors may develop Budd-Chiari syndrome (defined by hepatomegaly, jaundice and ascites) leading to a poor prognosis, tumors located in the middle segment usually present with right upper quadrant pain, or imitate biliary tract diseases, usually associated with better outcomes [11]. Tumor excision within the required oncological surgical margins may often involve extensive IVC repair and reconstruction, associated with increased postoperatively

This is an open-access article distributed under the terms of a Creative Commons Attribution-NonCommercial-ShareAlike 4.0 International Public License, which permits unrestricted use, adaptation, distribution and reproduction in any medium, non-commercially, provided the new creations are licensed under identical terms as the original work and the original work is properly cited. 
morbidity and higher mortality. The tumoral growing pace may be steeper or more rampant, showing expansive local invasion and metastasizes more frequently than previously believed [12]. Hematogenous metastases are more frequent, but in advanced stages, IVCL may also spread through the lymphatic system.

Multiple imaging techniques have been assessed and compared for the accuracy of preoperative diagnosis. However, it seems that magnetic resonance imaging (MRI) yields the highest accuracy for IVCL diagnosis, compared to contrast-enhanced computed tomography (CT) [13]. During the first diagnostic workup, a technique comprising of simultaneous contrast agent injection in both pedal veins has been described, using direct multidetector CT venography, with the great advantage of reaching the maximum vena cava enhancement. The results were comparable with conventional cavography, the gold standard for evaluating the IVC [14], similar achievements being reported for contrast-enhanced magnetic resonance (MR) venography [15].

Five-year disease-free survival and overall survival reported so far are $6 \%$ and $55 \%$, respectively [2]. Longterm survival is dependent of the extent and radicality of the surgery. In case recurrence is diagnosed, surgery remains a valid therapeutic option, being effective for controlling disease progression, possibly improving survival [16]. In case complete resection is impossible, combining debulking surgery with radiation therapy provides good palliation [17].

\section{Aim}

We hereby present a case of a patient with IVCL, with a number of particular therapeutic and surgical features. In addition, a review of the literature regarding treatment options and prognosis was performed.

\section{동 presentation}

A 61-year-old female patient without significant comorbidities presented for a check-up for diffuse abdominal pain. The biological evaluation did not reveal any pathological changes, but ultrasonography identified the presence of a right renal mass. Contrast-enhanced abdominal CT was performed and confirmed the diagnosis of a bulky right renal tumor. Chest CT did not reveal any

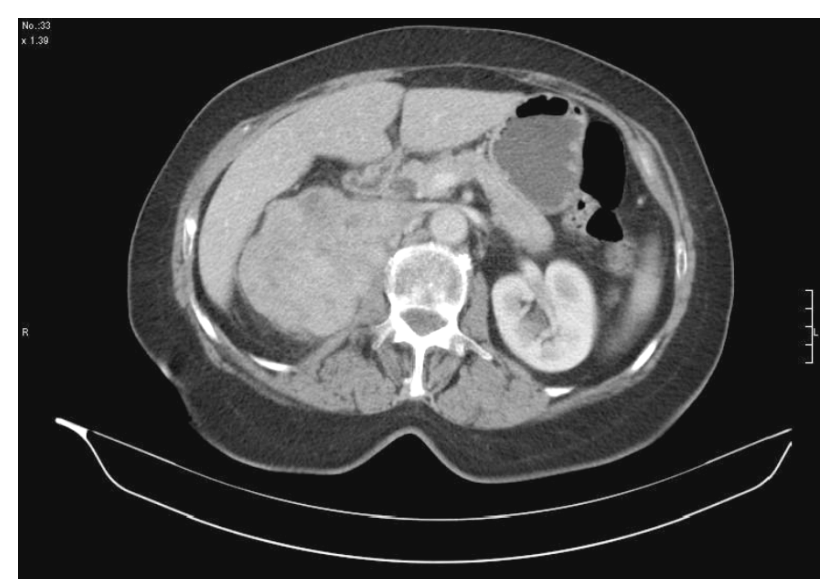

Figure 1 - Preoperative computed tomography: right renal bulky tumor. pulmonary metastasis, thus allowing the final staging as cT3aN0M0 (Figure 1). On the preoperative imaging evaluation, there was no evidence of the invasion of the vena cava, which was medially dislocated, but significant collateral circulation was present. Due to the characteristic appearance of the tumor at imaging scans, pre-operative biopsy was not performed. The patient signed the informed consent prior to publication of this case.

Following the preparation for standard transperitoneal right radical nephrectomy, the surgical intervention was performed in general anesthesia with orotracheal intubation by pararectal prolonged right subcostal approach. One of the main issues during the surgery was the difficult dissection of the renal pedicle, due to the significant collateral vessels and tumoral invasion. Due to this fact, the surgical strategy was modified. First, circumferential dissection of the tumor was performed. The dissection continued towards the renal hilum, where it was observed that the tumor encompassed the renal vessels and a significant segment of the IVC. Therefore, the next step was to isolate the IVC using vessel loops. During the medial dissection of the tumor, the injury of the infrarenal vena cava occurred. In order to perform hemostasis, rapid excision of the remaining tumor and further isolation of IVC was performed. A Satinsky clamp was placed on the lateral wall of the IVC and a suture was performed using Prolene 4/0. Declamping of the IVC was followed by repeating hemorrhage, thus the decision was taken to completely suture the infrarenal IVC in a transverse manner. The blood loss was $2.500 \mathrm{~mL}$ and transfusion was required. Postoperatively, the patient presented edema of the inferior limbs, which resolved by conservative approach in five days. Also, a transient kidney dysfunction was present for the first 72 hours.

The macroscopic analysis of the tumor specimen identified the presence of a tumor-adherent tubular vascular formation, which raised the suspicion of the vascular origin for the first time (Figure 2). Grossly, the surgical specimen measured 16.5/11/6 cm and was a block composed of kidney with perirenal fat, adrenal gland and fragment of vena cava. The tumor was located at the upper pole of the kidney, infiltrating the capsule and vena cava wall; it was well circumscribed and measured $9 \times 9 \times 6 \mathrm{~cm}$. Renal vessels and ureter were free of tumor. The cut surface was grey-white, firm, with foci of hemorrhage and necrosis.

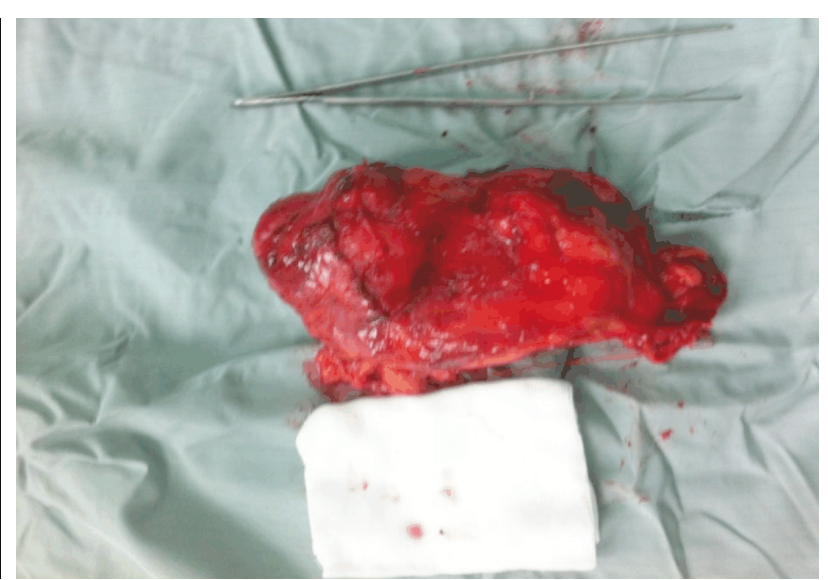

Figure 2 - The surgical specimen measured $16.5 / 11 / 6 \mathrm{~cm}$ and was a block composed of kidney with perirenal fat, adrenal gland and segment of vena cava. 
For positive and differential diagnosis, tumor fragments were collected, fixed in $10 \%$ neutral buffered formalin and sent to the Laboratory of Pathology, where they were included in paraffin, then sectioned at the microtome and stained with Hematoxylin-Eosin (HE). Some histological preparations were immunohistochemically stained using anti-alpha smooth muscle (anti- $\alpha$-SMA) (monoclonal mouse anti-human smooth muscle actin, clone 1A4, 1:100 dilution, Dako), and anti-cluster of differentiation 34 (anti-CD34) (monoclonal mouse anti-human CD34 Class II, clone QBEnd 10, 1:50 dilution, Dako) antibodies.

Microscopically, the tumor consisted of spindle cells with eosinophilic cytoplasm arranged in sheets and whorled, intersecting fascicles (Figure 3A). There was pronounced nuclear pleomorphism and a mitotic rate of 16 mitoses/ 10 high-power fields (HPFs) (field diameter was $0.5 \mathrm{~mm}$ ), also with atypical mitosis (Figure 3B). In some areas of the tumor, small areas of necrosis and inflammatory infiltrate have been identified (Figure 3C). The tumor cells were strongly positive for $\alpha$-SMA (Figure 3, D and E). CD34, by revealing the endothelium, showed an intense vascularization of the tumor, the vessels being formed mainly of arterioles, capillaries and venules, some of the capillaries being identified as vessels of angiogenesis (Figure 3F).
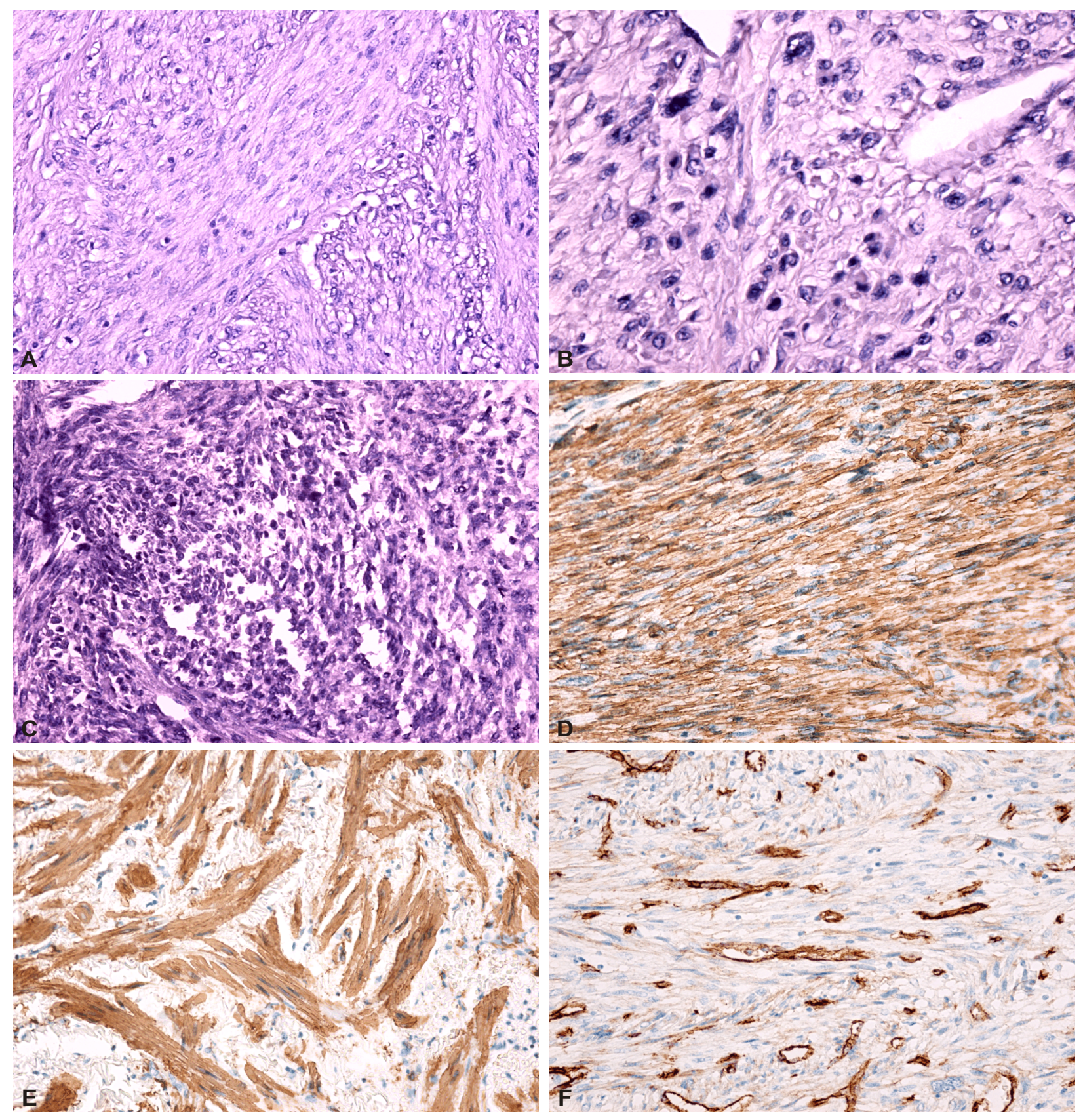

Figure 3 - (A) Histologically, the tumor consisted of spindle cells with eosinophilic cytoplasm arranged in sheets and whorled, intersecting fascicles; (B) There was pronounced nuclear pleomorphism and a mitotic rate of 16 mitoses/10 HPFs (field diameter was $0.5 \mathrm{~mm}$ ), also with atypical mitosis; (C) Area of tumor necrosis with a moderate intratumoral inflammatory infiltrate; (D) Tumor cells organized in layers, with positive reaction to $\alpha-S M A$; (E) Tumor cells organized in bundles with various orientations, with positive reaction to $\alpha-S M A ;(F)$ Tumor area with an increased microvascular density. HE staining: $(A$ and $C) \times 200 ;(B) \times 400$. Immunomarking with anti- $\alpha-S M A$ antibody: $(D$ and $E) \times 200$. Immunomarking with anti-CD34 antibody: $(F) \times 200$. HPFs: High-power fields; a-SMA: Alpha-smooth muscle actin; HE: Hematoxylin-Eosin; CD34: Cluster of differentiation 34. 
Macroscopic and microscopic aspects allow us to support the hypothesis that the origin of the tumor was the smooth muscles in the wall of the IVC.

During the four years of follow-up, no recurrent lesions were identified on the imaging, the patient being considered disease-free. Significant collateral venous circulation was described because of the suturing of the IVC (Figure 4).

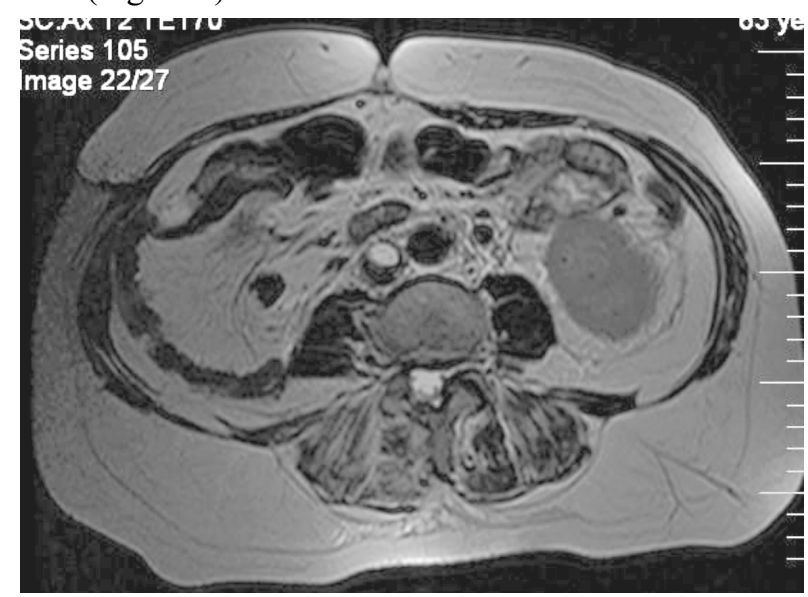

Figure 4 - The aspect of postoperative MRI: no recurrent lesions are detected, and numerous collateral venous circulation elements are present after suture of IVC. MRI: Magnetic resonance imaging; IVC: Inferior vena cava.

\section{ㅁ Discussions}

IVCLs are rare malignant tumors, often presenting very late with non-specific symptoms, like diffuse abdominal pain in the case hereby presented. A limited number of these cases have been described so far, occurring most frequently in middle-aged women. Only few of them have intracardiac propagation and surgery is rarely undertaken for their treatment [18].

A combination of imaging modalities, such as CT or MRI, is essential for treatment choice and planning [19]. The most characteristic CT sign for predicting the origin of IVCL is a poorly depictable IVC at the level where there is maximal contact with a retroperitoneal located tumor. The IVC origin of the tumor can be excluded in the presence of a negative embedded organ sign [20]. Knowledge of these CT characteristics represents the basis of a thorough preoperative planning. In a retrospective study [21], the imaging of primary tumor from 18 non-IVCLs and 19 IVCLs and follow-up imaging studies were reviewed; the authors found no significant difference between the imaging characteristics of these patients and the metastatic pattern of non-IVCL and IVCL. From a radiologist's perspective, non-IVCL behaves similar to IVCL. Survival of the patients with IVCL is no worse than of patients with leiomyosarcomatous lesions of other origin [22]. IVCL and non-IVCL exhibit similar outcomes in terms of postoperative course and survival [23].

In our case, there was no imaging evidence of the invasion of the vena cava, which appeared medially dislocated, but there was a significant collateral circulation. The tumor was located at the upper pole of the kidney, infiltrating the capsule and vena cava wall; it was well circumscribed and measured 9/9/6 cm. The macroscopic analysis of the tumor specimen from our patient identified the presence of a tumor-adherent tubular vascular formation, which raised the suspicion of the vascular origin for the first time.

When confronted with an imagistic finding, such as a tumor-like formation that occupies the IVC, differential diagnosis must be made with other pathologies that have an accentuated venous tropism and during their natural evolution might involve the vena cava. Renal cell carcinoma should always be considered when it comes to IVC tumoral extension. Between $5 \%$ and $15 \%$ of renal cell neoplasms develop a tumoral thrombus that penetrates the renal vein and can occupy the IVC up to the right atrium, in $1 \%$ of the cases [24]. Usually, the histopathological result of renal cell carcinoma is the most frequent scenario, especially when associated with a voluminous renal mass. However, as described in our case, other rare histological etiologies can be present. Another malignancy that can be held accountable for interesting the IVC is hepatocellular carcinoma, most commonly affecting the portal vein, but in up to $4 \%$ of the cases, it invades the IVC and extends to the right atrium, possibly causing pulmonary embolism [25]. An abdominal contrast-enhanced CT scan can differentiate between a pathology primarily emerging from the liver versus the kidney, as well as the final histological result. Regarding tumors that are most likely to have a benign evolution, cases of renal angiomyolipoma [26] and pheochromocytoma [27] have been reported to express an aggressive pattern and to extend into the IVC, especially if they are right-sided. From a radiological point of view, intraluminal tumor-like images can be the result of poor mixing of the contrast agent from the renal veins with the blood coming from the lower limbs. This effect is seen preponderantly in patients with congestive heart failure [28].

From a different perspective, IVCL should be differentiated from LMSs arising from surrounding structures, mainly from the kidney and the retroperitoneum. Renal LMSs develop from the renal structures that have smooth muscle layers, such as the renal capsule, pelvis and vein. The most frequent site is the renal vein [29], being also the most common location for extracaval LMSs, followed by renal capsule [30] and renal pelvis [31]. Fifteen percent of all soft tissue sarcomas are located in the retroperitoneum, $20 \%$ of them being LMSs. The primary curative treatment is represented by surgery, while radiation therapy and chemotherapy, although associated with surgery or stand-alone therapy in advanced cases, cannot establish the same outcomes and long-term survival rates as full tumoral excision [32]. In order to differentiate a primary IVCL from the fore mentioned sites, preoperatively contrastenhanced CT scan is required, as well as immunohistochemical staining of the specimen. In the presented case, the tumor was strongly positive for $\alpha$-SMA, as well as CD34, an endothelial marker, showing its vascular origin. With the renal vessels and collecting system free of disease, we can state that the primary site of this tumor is the IVC.

Despite recent research regarding different therapeutic strategies for IVCL, surgical resection with a tumor-free 
margin $(1 \mathrm{~cm})$ seems to be the approach with the highest probability to cure this disease. Unfortunately, only a small percentage of patients are eligible to undergo surgical intervention. The optimal surgical management remains a controversy [19]. The lack of studies assessing large series of IVCL treated in the same center and lack of longterm follow-up lead to uncertainties regarding the best treatment algorithm [33]. Preoperative external-beam radiation may facilitate a resection with negative surgical margins [34]. Also, cross-sectional imaging, by allowing to establish the exact location and IVC extension of the tumor, plays a vital role in determining the resectability and planning the surgical approach [35].

A review of the literature including 142 patients [33] showed approximately similar percentages of tumors originating from the three segments of IVC. In 49 cases, the tumor arose from the infrarenal segment of the IVC. In 59 patients, the IVCL had the origin in the segment between the renal pedicle and hepatic veins, whereas in 34 patients the tumor was located between the hepatic veins and the right atrium. In 82 cases, the tumor was radically resected, leading to improved long-term survival (27.9\% at five years and $14.2 \%$ at 10 years). The survival of the patients with middle segment IVCL was significantly higher as compared to lower-segment tumor $(48.3 \%$ vs. $9.3 \%$ at five years). Good prognosis was associated with the presence of abdominal pain and the lack of a palpable tumor at diagnosis. Despite the high recurrence rate $(52.4 \%$ at two years), extensive radical resection of IVCL was the main predictor for a potentially curative approach.

For our patient, the lateral wall of the IVC was initially clamped and the suture of the vein wall was performed, but the hemorrhage reappeared and the transverse suture of the infrarenal portion of IVC was performed as the only solution for vital hemorrhagic risk.

Although the IVCL is surgically removed, this does not necessarily guarantee a long-term survival for the patient. Furthermore, complementary therapies, such as radio- and chemo-therapy often prove insufficient oncological control. The recurrence of the tumor after previous initial surgical resection is commonly diagnosed and occurs in the majority of the patients [36]. As a particularity, in our case, during a follow-up of four years, no recurrence of the tumor was identified, in comparison with other reported cases who underwent more than 20 surgical reinterventions after the primary surgery.

The optimal management of the IVC after tumor resection is still a subject under debate. Primary repair, ligation and IVC reconstruction have been utilized with different success rates [37]. The need of vascular reconstruction is not always mandatory [38]. The necessity to perform a large resection for a primary IVCL requires the segmental excision of IVC in some cases. Reconstruction of the IVC is not always necessary when the tumor is located below the level of the hepatic veins [34]. Even in the occurrence of lower-extremity edema secondary to the ligation of the IVC, this adverse situation is well tolerated. Other common post-operative complications are represented by acute renal failure, which is usually transient, and chylous leak, which can be managed with internal or external drainage.
Intraoperatively, in our case, an extremely abundant venous collateral circulation was identified. Postoperatively, our patient presented edema of the inferior limbs, resolved by conservative approach in five days.

In a study on three patients [6], the mean age at diagnosis of IVCL was 60.3 years (range 43-78 years), while the mean tumor size was $12.2 \mathrm{~cm}$. The authors reported a mean operative time of 320 minutes and a mean blood loss of $1300 \mathrm{~mL}$. The average length of hospital stay for these patients was 8.67 days, with a maximum of 12 days. Our patient experienced $2500 \mathrm{~mL}$ intraoperative blood loss, which was compensated by transfusion.

When performed, the reconstruction of an IVC defect after surgical resection can lead to significant postoperative complications, such as venous thrombosis or infection of the graft [39]. The reconstruction can be performed using autologous venous grafts from the patient's own left internal jugular and left external iliac vein. The two veins are incised along their axis and are sutured together to form a wider tube graft to match the IVC defect [39].

A retrospective review of patients diagnosed with IVCL and treated during a 10 -year period showed that neoadjuvant radiotherapy, followed by extensive surgical resection led to complete local control. The use of superficial femoral vein for IVC reconstruction was proven to be safe, with low associated morbidity. Longterm follow-up yielded good results, with the authors reporting that all patients were alive at a median followup of 37 months [40].

Although initial results of surgical resection of IVCL are encouraging, the majority of patients experience recurrence. However, as reported by several authors, long-term survival is possible [41]. The predictors of survival after surgical excision of IVCL include margin status, tumor size and the radicality of the resection [2]. On the other hand, in a study of 218 patients enrolled into The International Registry of IVCL, from which 120 patients underwent a radical resection of the IVCL, an extended venous resection in IVCL did not influence neither local recurrence rate nor long-term outcome [42].

There were 143 reports of IVCL in Japan. In $31 \%$ of them, IVC was not reconstructed [43]. In a series of five patients with IVCL treated at two Chinese centers, the authors report no IVC related postoperative complications, although the surgeries included multiorgan resection, without IVC reconstruction [19].

The first case of IVCL, involving all three segments of the abdominal IVC (infrarenal, suprarenal and retrohepatic vena cava) along with right kidney, right adrenal as well as right hepatic vein and left renal vein, treated without IVC reconstruction, was described in a study [44]. IVCL was resected completely and despite its extent and concomitant involvement of surrounding organs, it had a favorable response combining prolongation of survival and satisfactory quality of life.

\section{a Conclusions}

IVCL is a treatable malignancy, but remains a challenge for surgeons. Imagistic studies need to be performed preoperatively in order to correctly assess its extension 
and properly plan the surgery. Despite recent research regarding the best therapeutic strategy for IVCL and the high rate of recurrence, surgical resection appears the only potentially curative treatment. A multidisciplinary approach including surgical oncologists and vascular surgeons ensures maximal resection with functional reconstruction to achieve the best patient outcomes. Perioperative planning, coordination and adherence to oncological techniques are critical.

\section{Conflict of interests}

Authors declare that there is no conflict of interests.

\section{References}

[1] Kapoor R, Bansal A, Sharma SC. Leiomyosarcoma of inferior vena cava: case series of four patients. J Cancer Res Ther 2015, 11(3):650. https://doi.org/10.4103/0973-1482.138029 PMID: 26458620

[2] Wachtel H, Gupta M, Bartlett EK, Jackson BM, Kelz RR, Karakousis GC, Fraker DL, Roses RE. Outcomes after resection of leiomyosarcomas of the inferior vena cava: a pooled data analysis of 377 cases. Surg Oncol, 2015, 24(1): 21-27. https://doi.org/10.1016/j.suronc.2014.10.007 PMID: 25433957

[3] Drukker L, Alberton J, Reissman P. Leiomyosarcoma of the inferior vena cava: radical surgery without vascular reconstruction. Vasc Endovascular Surg, 2012, 46(8):688-690. https:// doi.org/10.1177/1538574412460102 PMID: 22983880

[4] Perl L, Virchow R. Ein Fall von Sarkom der Vena cava inferior [Leiomyosarcoma of inferior vena cava]. Virchows Arch Pathol Anat, 1871, 53(1):378-383. https://doi.org/10.1007/BF01957198

[5] Alexander A, Rehders A, Raffel A, Poremba C, Knoefel WT, Eisenberger CF. Leiomyosarcoma of the inferior vena cava: radical surgery and vascular reconstruction. World J Surg Oncol, 2009, 7:56. https://doi.org/10.1186/1477-7819-7-56 PMID: 19558690 PMCID: PMC2710329

[6] Alkhalili E, Greenbaum A, Langsfeld M, Marek J, Rana MA Glew R, Nir I. Leiomyosarcoma of the inferior vena cava: a case series and review of the literature. Ann Vasc Surg, 2016, 33:245-251. https://doi.org/10.1016/j.avsg.2015.10.016 PMID: 26802297

[7] Fernandez HT, Kim PTW, Anthony TL, Hamman BL, Goldstein RM, Testa G. Inferior vena cava reconstruction for leiomyosarcoma of zone I-III requiring complete hepatectomy and bilateral nephrectomy with autotransplantation. J Surg Oncol, 2015, 112(5):481-485. https://doi.org/10.1002/jso.24 041 PMID: 26356493

[8] Guillou L, Coindre JM, Bonichon F, Nguyen BB, Terrier P, Collin F, Vilain MO, Mandard AM, Le Doussal V, Leroux A, Jacquemier J, Duplay H, Sastre-Garau X, Costa J. Comparative study of the National Cancer Institute and French Federation of Cancer Centers Sarcoma Group grading systems in a population of 410 adult patients with soft tissue sarcoma. J Clin Oncol, 1997, 15(1):350-362. https://doi.org/10.1200/ JCO.1997.15.1.350 PMID: 8996162

[9] Taylor RW, Sylwestrowicz T, Kossakowska AE, Urbanski SJ, Minuk GY. Leiomyosarcoma of the inferior vena cava presenting as Budd-Chiari syndrome. Liver, 1987, 7(4):201-205. https:// doi.org/10.1111/j.1600-0676.1987.tb00343.x PMID: 3683091

[10] Lozinguez O, Emmerich J, Fornes P, Fiessinger JN. [Leiomyosarcoma of the inferior vena cava]. Presse Med, 1998, 27(18): 855-857. PMID: 9767869

[11] Biró ZJ, Kalmár Nagy K, Fehér E, Gömöri E, Horváth OP [Surgical treatment of primary leiomyosarcoma of the inferior vena cava]. Magy Seb, 2009, 62(2):83-86. https://doi.org/10. 1556/MaSeb.62.2009.2.6 PMID: 19386569

[12] Cinà CS, Riccioli V, Passanisi G, Musumeci G, Loreto C, Castorina S. Computerized tomography and 3-D rendering help to select surgical strategy in leiomyosarcoma of the inferior vena cava. Updates Surg, 2013, 65(4):283-288. https://doi. org/10.1007/s13304-013-0225-0 PMID: 23888374

[13] Mönig SP, Gawenda M, Erasmi H, Zieren J, Pichlmaier H. Diagnosis, treatment and prognosis of the leiomyosarcoma of the inferior vena cava. Three cases and summary of published reports. Eur J Surg, 1995, 161(4):231-235. PMID: 7612763
[14] Ulla M, Kohan A, Pekolj J, Isola M, Garcia-Mónaco R. Direct 64-row MDCT venography in the diagnosis of an inferior vena cava leiomyosarcoma. Abdom Imaging, 2011, 36(3):333-336. https://doi.org/10.1007/s00261-010-9630-8 PMID: 20585777

[15] Huang J, Liu Q, Lu JP, Wang F, Wang L, Jin AG. Primary intraluminal leiomyosarcoma of the inferior vena cava: value of MRI with contrast-enhanced MR venography in diagnosis and treatment. Abdom Imaging, 2011, 36(3):337-341. https:// doi.org/10.1007/s00261-010-9656-y PMID: 20981422

[16] Sulpice L, Rayar M, Levi Sandri GB, de Wailly P, Henno S, Turner K, Lesimple T, Boudjema K, Meunier B. Leiomyosarcoma of the inferior vena cava. J Visc Surg, 2016, 153(3): 161-165. https://doi.org/10.1016/j.jviscsurg.2015.11.002 PMID: 26711879

[17] Yadav R, Kataria K, Mathur SR, Seenu V. Leiomyosarcoma of inferior vena cava: a case series of four cases. Indian $\mathrm{J}$ Pathol Microbiol, 2012, 55(1):83-85. https://doi.org/10.4103/ 0377-4929.94865 PMID: 22499308

[18] Matić P, Vučurević G, Babić S, Tanasković S, Lozuk B Sagić D, Radak D. Intracardiac extension of the inferior vena cava leiomyosarcoma with Budd-Chiari syndrome presentation: a case report. Srp Arh Celok Lek, 2015, 143(1-2):71-73. https://doi.org/10.2298/sarh1502071m PMID: 25845255

[19] Jiang $H$, Wang $Y X$, Li B, Jiang YY, Miao CL, Liao DX, Zhao RH, Luo $\mathrm{CH}$. Surgical management of leiomyosarcoma of the inferior vena cava. Vascular, 2015, 23(3):329-332. https:// doi.org/10.1177/1708538114547755 PMID: 25171923

[20] Webb EM, Wang ZJ, Westphalen AC, Nakakura EK, Coakley FV, Yeh BM. Can CT features differentiate between inferior vena cava leiomyosarcomas and primary retroperitoneal masses? AJR Am J Roentgenol, 2013, 200(1):205-209. https://doi.org/ 10.2214/AJR.11.7476 PMID: 23255763

[21] Cooley CL, Jagannathan JP, Kurra V, Tirumani SH, Saboo SS, Ramaiya $\mathrm{NH}$, Shinagare AB. Imaging features and metastatic pattern of non-IVC retroperitoneal leiomyosarcomas: are they different from IVC leiomyosarcomas? J Comput Assist Tomogr, 2014, 38(5):687-692. https://doi.org/10.1097/RCT.0000000 000000097 PMID: 24834891

[22] Hines OJ, Nelson S, Quinones-Baldrich WJ, Eilber FR. Leiomyosarcoma of the inferior vena cava: prognosis and comparison with leiomyosarcoma of other anatomic sites. Cancer, 1999, 85(5):1077-1083. https://doi.org/10.1002/(SICI) 1097-0142(19990301)85:5<1077::AID-CNCR10>3.0.CO;2-0 PMID: 10091791

[23] Illuminati G, Pizzardi G, Calio' F, Pacilè MA, Masci F, Vietri F. Outcome of inferior vena cava and noncaval venous leiomyosarcomas. Surgery, 2016, 159(2):613-620. https://doi.org/10. 1016/j.surg.2015.08.026 PMID: 26435438

[24] Noguchi K, Hori D, Nomura Y, Tanaka H. Renal cell carcinoma with tumor-thrombus extension into the right ventricle. Ann Vasc Dis, 2012, 5(3):376-380. https://doi.org/10.3400/avd.cr. 11.00067 PMID: 23555539 PMCID: PMC3595859

[25] Li W, Wang Y, Gao W, Zheng J. HCC with tumor thrombus entering the right atrium and inferior vena cava treated by percutaneous ablation. BMC Surg, 2017, 17(1):21. https:// doi.org/10.1186/s12893-017-0217-y PMID: 28245832 PMCID: PMC5331644

[26] Tan YS, Yip KH, Tan PH, Cheng WS. A right renal angiomyolipoma with IVC thrombus and pulmonary embolism. Int Urol Nephrol, 2010, 42(2):305-308. https://doi.org/10.1007/ s11255-009-9612-3 PMID: 19609707

[27] Kota SK, Kota SK, Jammula S, Meher LK, Modi KD. Pheochromocytoma with inferior vena cava thrombosis: an unusual association. J Cardiovasc Dis Res, 2012, 3(2):160-164. https://doi.org/10.4103/0975-3583.95375 PMID: 22629039 PMCID: PMC3354464

[28] Molina M, Schiappacasse G, Labra A. Tumors that invade the inferior vena cava: an illustrative review of the main imaging features on computed tomography and magnetic resonance. Rev Chil Radiol, 2016, 22(1):39-46. https://doi. org/10.1016/j.rchira.2016.02.005

[29] Novak M, Perhavec A, Maturen KE, Pavlovic Djokic S, Jereb S, Erzen D. Leiomyosarcoma of the renal vein: analysis of outcome and prognostic factors in the world case series of 67 patients. Radiol Oncol, 2016, 51(1):56-64. https://doi.org/ 10.1515/raon-2016-0051 PMID: 28265233 PMCID: PMC 5330168 
[30] Beardo P, Ledo MJ, Luis RCJ. Renal leiomyosarcoma. Rare Tumors, 2013, 5(3):e42. https://doi.org/10.4081/rt.2013.e42 PMID: 24179654 PMCID: PMC3804817

[31] Minami H, Ueki O, Tanaka T, Nishida H, Hashimoto $T$, Kawaguchi K. Case of leiomyosarcoma of the renal pelvis. Int J Urol, 2004, 11(2):122-124. https://doi.org/10.1111/j.14 42-2042.2004.00747.x PMID: 14706019

[32] Mantas D, Garmpis N, Polychroni D, Garmpi A, Damaskos C, Liakea A, Sypsa G, Kouskos E. Retroperitoneal sarcomas: from diagnosis to treatment. Case series and review of the literature. G Chir, 2020, 41(1):18-33. PMID: 32038009

[33] Mingoli A, Feldhaus RJ, Cavallaro A, Stipa S. Leiomyosarcoma of the inferior vena cava: analysis and search of world literature on 141 patients and report of three new cases. J Vasc Surg, 1991, 14(5):688-699. https://doi.org/10.1067/ mva.1991.30426 PMID: 1942380

[34] Daylami R, Amiri A, Goldsmith B, Troppmann C, Schneider PD, Khatri VP. Inferior vena cava leiomyosarcoma: is reconstruction necessary after resection? J Am Coll Surg, 2010, 210(2):185190. https://doi.org/10.1016/j.jamcollsurg.2009.10.010 PMID: 20113938

[35] Naphade PS, Raut AA, Hira P, Vaideeswar P, Vadeyar H. Leiomyosarcoma of the inferior vena cava. Arch Iran Med, 2014, 17(5):383-387. https://doi.org/ PMID: 24784870

[36] Rascanu C, Duran M, Grabitz K, Weis-Müller B, Sandmann W. Successful surgical management of a recurrent leiomyosarcoma of the inferior vena cava. Vasa, 2011, 40(1):69-72. https://doi. org/10.1024/0301-1526/a000072 PMID: 21283976

[37] Mastoraki A, Leotsakos G, Mastoraki S, Papanikolaou IS Danias N, Smyrniotis V, Arkadopoulos N. Challenging diagnostic and therapeutic modalities for leiomyosarcoma of inferior vena cava. Int J Surg, 2015, 13:92-95. https://doi.org/10.1016/j. ijsu.2014.11.051 PMID: 25489949
[38] Slimane M, Yahia NB, Bouaziz H, Bouzaine H, Benhassouna J, Dhieb TB, Hechiche M, Gammoudi A, Rahal K. Inferior vena cava leiomyosarcoma: vascular reconstruction is not always mandatory. Pan Afr Med J, 2016, 24:287. https://doi.org/10. 11604/pamj.2016.24.287.8912 PMID: 28154642 PMCID: PMC5267787

[39] Soejima Y, Matsumoto T, Shirabe K, Maehara Y. Tube cavoplasty using autologous vein grafts for resected inferior vena cava reconstruction. Surg Today, 2013, 43(4):452-455. https://doi.org/10.1007/s00595-012-0427-y PMID: 23188386

[40] Munene G, Mack LA, Moore RD, Temple WJ. Neoadjuvant radiotherapy and reconstruction using autologous vein graft for the treatment of inferior vena cava leiomyosarcoma. J Surg Oncol, 2011, 103(2):175-178. https://doi.org/10.1002/jso.21 798 PMID: 21259253

[41] Madhavan S, Junnarkar SP, Koh NWC, Shelat VG. Inferior vena cava leiomyosarcoma in an octogenerian. Ann Hepatobiliary Pancreat Surg, 2019, 23(3):274-277. https://doi.org/ 10.14701/ahbps.2019.23.3.274 PMID: 31501817 PMCID: PMC6728244

[42] Mingoli A, Sapienza P, Cavallaro A, Di Marzo L, Burchi C, Giannarelli D, Feldhaus RJ. The effect of extend of caval resection in the treatment of inferior vena cava leiomyosarcoma. Anticancer Res, 1997, 17(5B):3877-3881. PMID: 9427796

[43] Yo T, Taoka R, Hanasaki T, Nakanishi Y, Togo Y, Suzuki T, Higuchi Y, Zozumi M, Hirota S, Kanematsu A, Nojima M, Yamamoto S. [Leiomyosarcoma of the inferior vena cava: a case report and review]. Hinyokika Kiyo, 2014, 60(3):115119. PMID: 24759496

[44] Lygidakis NJ, Bhagat AD, Sharma SK, Kefalourous H, Porfiris T, Grigorakos L, Vrachnos $P$, Mihalopoulos A, Bleta A, Bodozoglou N, Voulgari K, Vlachos L. Leiomyosarcoma of the inferior vena cava - an unusual case. Hepatogastroenterology, 2007, 54(75):710-715; discussion 716-717. PMID: 17591047

\section{Corresponding author}

Iulia Mădălina Andraş, Assistant Professor, MD, PhD, Fellow of the European Board of Urology (FEBU), Department of Urology, luliu Haţieganu University of Medicine and Pharmacy, Municipal Clinical Hospital, 11 Tăbăcarilor Street, 400139 Cluj-Napoca, Romania; Phone +40744-374 996, e-mail: dr.iuliaandras@gmail.com 